\title{
Sinonasal Teratocarcinosarcoma
}

\author{
Shaikh S ${ }^{1}$, Fernandes $\mathrm{G}^{2}$, Rojekar $\mathrm{A}^{3}$, Joshi $\mathrm{A}^{4}$ \\ ${ }^{1}$ Dr. Shaikh S, $3^{\text {rd }}$ year Resident, ${ }^{2}$ Dr. Fernandes G, Associate Professor, ${ }^{3}$ Dr Rojekar A, Assistant Professor ${ }^{3},{ }^{4}$ Dr. Joshi \\ A, Professor \& Head, all authors are affiliated with Department of Pathology, Seth G.S Medical College \& K.E.M \\ Hospital, Mumbai, India
}

Address for Correspondence: Dr Gwendolyn Fernandes, C-802, Swayam, Poonam Gardens, Mira Road, Thane, Maharashtra. E-mail: drgwenfern@yahoo.co.in, $\underline{\text { drgwenfern@ gmail.com }}$

\begin{abstract}
Sinonasal Teratocarcinosarcoma (SNTCS) is an extremely rare and unusual malignant neoplasm of the sinonasal tract with an aggressive behaviour. Till date, there are less than a hundred cases reported in literature. A 52 year old patient presented with a large, reddish brown, right sided nasal mass with epistaxis since 1 month. Histopathology revealed a highly malignant tumor with diverse histomorphological features and was labelled SNTCS. These unique tumors show diverse histomorphological features of a carcinosarcoma with teratoid elements. Immunohistochemistry was positive for cytokeratin, vimentin, GFAP, NSE, S-100. Chemotherapy with cisplatin and etoposide was given followed by a debulking surgery. The patient is well seven months after diagnosis and scheduled for radiotherapy. Diagnosis of SNTCS requires an adequate representative biopsy and extensive sampling on Histopathology. Treatment required included surgery, chemotherapy and radiotherapy.
\end{abstract}

Keywords: Sinonasal teratocarcinoma, sinonasal tumors, unusual tumors

\section{Introduction}

SNTCS is a very rare and unusual malignant neoplasm of the sinonasal tract with a heterogenous morphology and an aggressive behaviour. Till date, there are less than 100 cases reported in literature [1]. Histologically, SNTCS is a unique tumor comprising varying combinations of benign and malignant epithelial, mesenchymal and neuroectodermal elements [2,3]. This tumor is a combination of a carcinosarcoma and a teratoma [4] Extensive excision and sampling for histopathology is required since this tumor has diverse morphological features varying from benign to malignant and varied components $[3,6]$.

\section{Case Report}

A 52 year old patient presented with a right nasal mass with epistaxis since 1 month. The patient also had symptoms of nasal obstruction, pain, headache and anosmia. On clinical examination, a 5 x $4 \mathrm{~cm}$, reddish brown mass which bled on touch was seen. Detailed radiological examination was done and biopsy was taken.

CT and MRI showed a large, non-enhancing or heterogeneously enhancing soft tissue mass filling the nasal cavity, nasopharynx and sinuses, and were suggestive of a benign lesion.

The biopsy received measured $2.5 \times 1.5 \mathrm{~cm}$ and was a single greyish-white mass. Histopathological examination revealed fibrovascular tissue with dilated vascular channels which was not diagnostic. Nasal mass excision with right uncinectomy was done and the mass sent for Histopathology. We received multiple, soft fleshy, friable yellowish brown bits of tissue. 

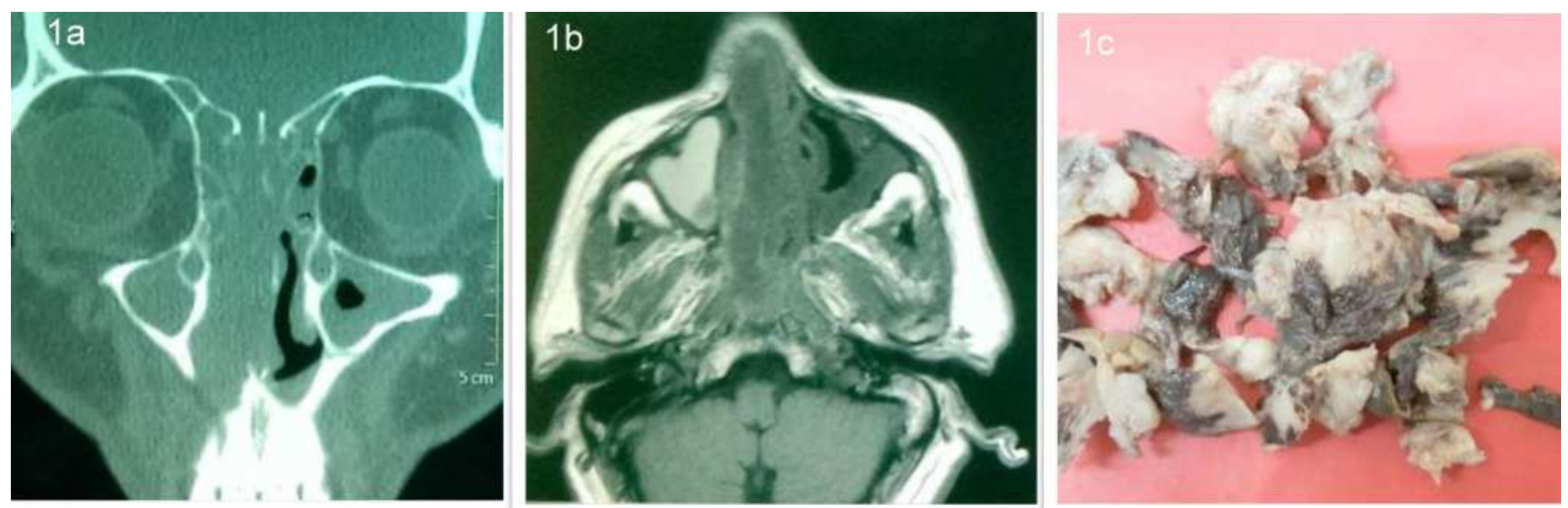

Fig. 1a- CT- large, non enhancing soft tissue density mass lesion seen filling the right maxillary sinus and extending beyond.

Fig. 1b- MR- brain and paranasal sinuses - heterogeneously enhancing soft tissue density mass in the nasal cavity.

Fig. 1c- Gross- multiple, soft, friable yellowish brown bits with areas of hemorrhage.

Microscopic examination revealed a tumor with diverse morphology which varied from a benign to highly malignant admixture of epithelial, mesenchymal and neuroepithelial elements. The epithelial component varied from benign glandular epithelium to malignant olfactory epithelium. The mesenchymal component comprised of benign to malignant spindle shaped cells in a myxoid stroma. Neuroepithelial components comprised of nests of small blue malignant cells.
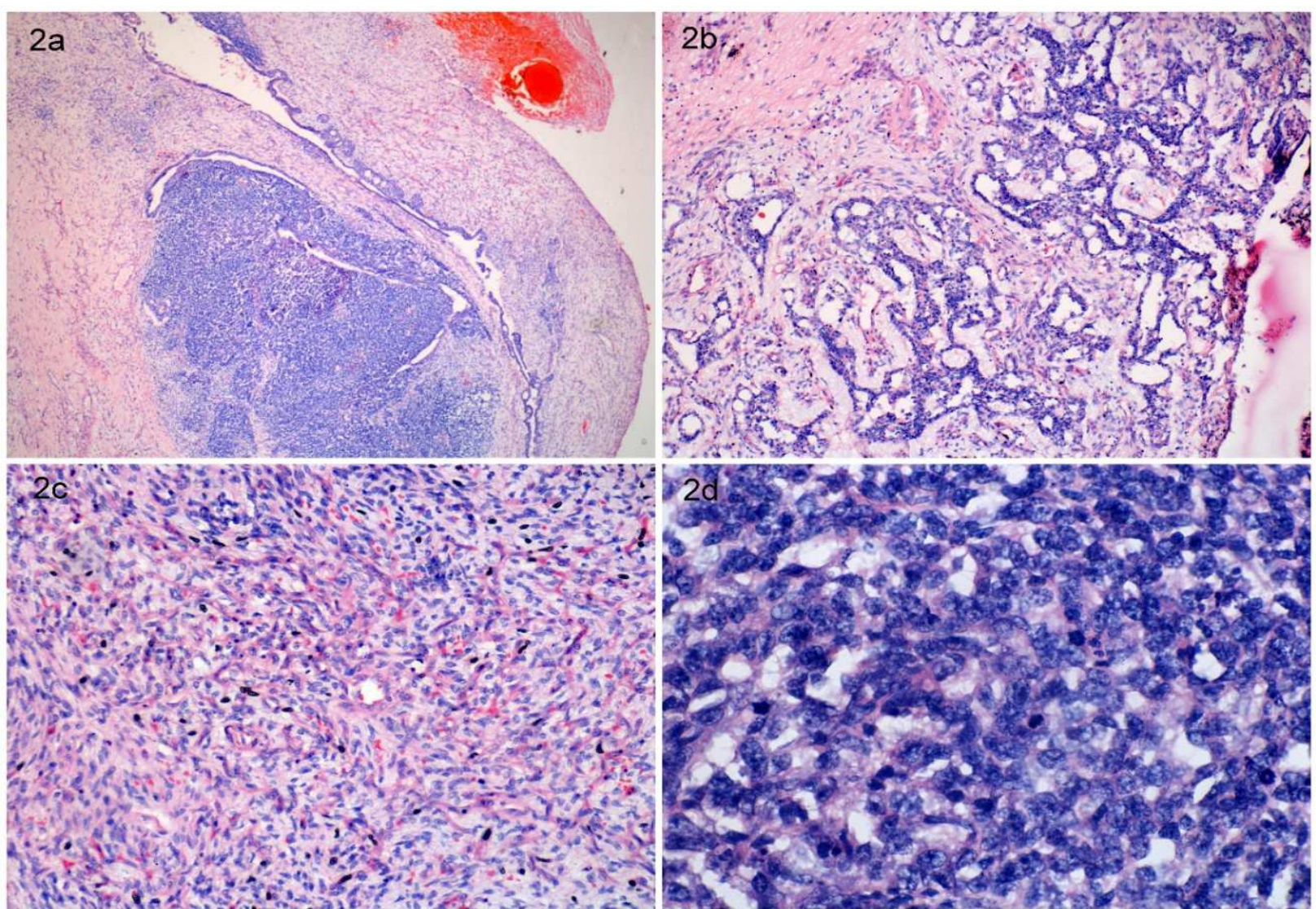

Fig. 2a-Scanner view: various components of the tumor showing heterogeneous admixture of epithelial components, mesenchymal and neuroepithelial component (H \& E x 40).

Fig. 2b- Glandular formations lined by benign cuboidal to columnar epithelium (H \& E x 100).

Fig. 2c- Spindle cell mesenchyme showing mild to moderate atypia (H \& E x 400).

Fig. 2d- Primitive neuroepithelial component composed of malignant small round blue cells with hyperchromatic nuclei and minimal cytoplasm (H \& E x 400). 
Occasional rosettes, mitotic figures and necrosis were seen. Fetal appearing clear squamous cells and organoid structures were not seen. On Immunohistochemistry, the epithelial components stained positively with cytokeratin, mesenchymal components were positive for vimentin, while S-100 showed focal cytoplasmic and nuclear positivity. The neuroepithelial component was positive for NSE and GFAP. Synatophysin, chromogranin, CD 99, CD 34 were negative in the tumor. A diagnosis of SNTCS was made.
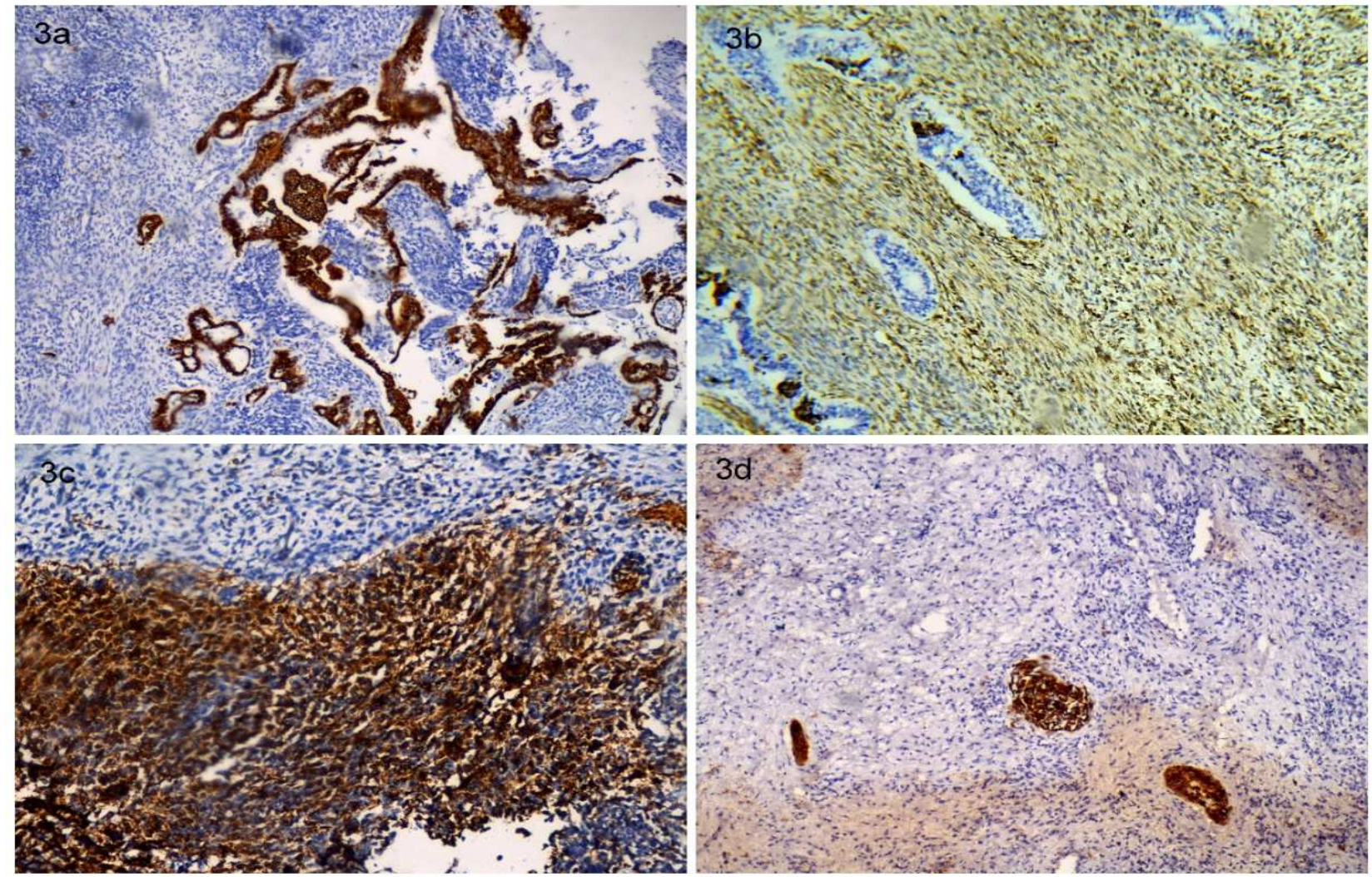

Fig.-3: IHC - 3a-CK positivity seen in epithelial component, 3b-Vimentin positivity seen in mesenchymal component, 3c-NSE positivity seen in neuroepithelial component, 3d-GFAP positivity seen in neuroepithelial component

The patient was given two cycles of chemotherapy with cisplatin and etoposide followed by debulking surgery and is due for two cycles of radiotherapy. He is now well and has resumed normal duties, seven months after diagnosis.

\section{Discussion}

SNTCS is an infrequent, aggressive and highly malignant neoplasm of the sinonasal region which is a combination of a teratoma and a carcinosarcoma comprising of benign to malignant epithelial components, mesenchymal components and neuroectodermal components [2,5]. Till date, less than a hundred cases have been reported in literature [1]. It was first described by Shanmugaratnam et al. but Hefner and Hyams first coined the term 'Teratocarcinosarcoma' [2,3]. This tumor is known to occur in the sinonasal tract. However, few cases of extranasal teratocarcinosarcomas have been reported in nasopharynx, orbit, posterior pharyngeal wall and tongue [6]. These tumors are usually seen in age group above 50 years but cases have been described from 18 to 79 years and even neonates [7]. A strong male preponderance is seen with male to female ratio of $7: 1$. $[1,3,7]$. The histogenesis of these tumors is not well known but they are believed to arise from immature pluripotent cells sequestered in the sinonasal tract which gives rise to divergent differentiation [10].

Clinically, SNTCS usually presents with recurrent epistaxis $(53.52 \%)$ and nasal obstruction $(61.97 \%)$ and are seen as reddish-brown, friable masses with necrotic areas which bleed on touch [11].

On histological examination, these tumors show diverse histomorphology of carcinosarcoma with teratoid elements [1]. Varying combinations of benign to 
malignant epithelial, mesenchymal and immature neuroectodermal components are seen as was in our case [10]. Our case showed benign glands, foci of adenocarcinoma, benign to malignant spindle cell proliferation and immature neuroepithelium with rosettes. Foetal appearing clear squamous cells and organoid structures were not seen which features of teratomas are.

Immunohistochemistry varies according to the histological cell type; epithelial components are CK and EMA positive; neuroepithelial components are NSE, CD99, GFAP and S-100 protein positive; mesenchymal components are positive for vimentin and myogenic markers [3]. Our case was CK positive in the epithelial areas, NSE, GFAP in the neuroblastoma areas and vimentin was positive in the mesenchymal component. S-100 showed focal positivity, while synatophysin, chromogranin, CD 99, CD 34 were all negative in the tumor.

Carcinosarcoma is an important differential diagnosis and it differs from SNTCS as it consist of single malignant epithelial and a single malignant mesenchymal component, whereas SNTCS has many such components [13]. Another differential diagnosis is olfactory neuroblastoma with divergent differentiation but this tumor does not show presence of overt epithelial differentiation and truemesenchymal components [6].

Treatment for this aggressive tumor includes radical excision followed by radiotherapy and chemotherapy $[6,10]$. Our patient received chemotherapy with cisplatin and etoposide followed by debulking surgery of the nasal mass and is currently posted for radiotherapy. He has resumed routine life now, seven months after initial diagnosis.

As SNTCS has diverse histomorphogical features, a small superficial biopsy may not be representative of this lesion and an accurate diagnosis may not be possible. The initial biopsy of this case was not diagnostic but histopathology of the excised nasal mass revealed the complete picture and the diagnosis was made.

\section{Conclusion}

Sinonasal teratocarcinosarcomas are rare, aggressive tumors and difficult to diagnose as they have diverse range of histomorphological features varying from benign to malignant. Hence, diagnosis of this lesion requires an adequate, representative biopsy including all components and thorough histopathological examination. Treatment required included surgery, chemotherapy and radiotherapy.

Funding: Nil, Conflict of interest: None initiated, Permission from IRB: Yes

\section{References}

1. Agrawal N, Chintagumpala M, Hicks J, Eldin K, Paulino AC. Sinonasal teratocarcinosarcoma in an adolescent male. J Pediatr Hematol Oncol. 2012; 34(7):e30.doi:10.1097/MPH.0b013e318266baa8

2. Heffner DK, Hyams VJ. Teratocarcinosarcoma (malignant teratoma?) of the nasal cavity and paranasal sinuses A clinicopathologic study of 20 cases. Cancer [Internet]. 1984 May 15 [cited 2016 Nov 30];53(10):2140-54.

3. Shanmugaratnam K, Kunaratnam N, Chia KB, Chiang GS, Sinniah R. Teratoid carcinosarcoma of the paranasal sinuses. Pathology [Internet]. 1983 Oct [cited 2016 Nov 30];15(4):413-9.

4. Wei S, Carroll W, Lazenby A, Bell W, Lopez R, Said-Al-Naief N. Sinonasal teratocarcinosarcoma: report of a case with review of literature and treatment outcome. Ann DiagnPathol. 2008 Dec;12(6):415-25. doi: 10.1016/j.anndiagpath.2007.05.003. Epub 2007 Oct 24.

5. Chakraborty S, Chowdhury AR, Bandyopadhyay G. Sinonasal teratocarcinosarcoma: Case report of an unusual neoplasm. J Oral Maxillofac Pathol. 2016; 20(1):147-50.doi:10.4103/0973-029X.180979

6. Fatima SS, Minhas K, Din NU, Fatima S, Ahmed A, Ahmad Z. Sinonasal teratocarcinosarcoma: a clinicopathologic and immunohistochemical study of 6 cases. Ann Diagn Pathol 2013; 17(4):313-8.doi: 10.1016/j.anndiagpath.2013.01.003. Epub 2013 Feb 23.

7. Rotenberg B, El-Hakim H, Lodha A, MacCormick A, Ngan BY, Forte V. Nasopharyngeal teratocarcinosarcoma. Int $\mathrm{J}$ Pediatr Otorhinolaryngol [Internet]. 2002 Feb 1 [cited 2016 Nov 30];62(2):15964. 
8. Crazzolara R, Puelacher W, Ninkovic M, Zelger B, Buchberger W, Meister B, et al. Teratocarcinosarcoma of the oral cavity. Pediatr Blood Cancer. 2004 Nov;43(6):687-91. doi:10.1002/pbc.20139

9. Carrizo F, Pineda-Daboin K, Neto AG, Luna MA. Pharyngeal teratocarcinosarcoma: review of the literature and report of two cases. Ann Diagn Pathol. 2006; 10(6):339-42.

doi:10.1016/j.anndiagpath.2006.03.006

10. Kane S V, Karpate AA, Bal M, Juvekar SL, Pai PS. Chemotherapy-induced neuronal maturation in sinonasal teratocarcinosarcoma--a unique observation. Head Neck Pathol. 2009; 3:31-6.doi: 10.1007/s12105008-0094-X. Epub 2008 Dec 4.
11. Takasaki K, Sakihama N, Takahashi H. A case with sinonasal teratocarcinosarcoma in the nasal cavity and ethmoid sinus. Eur Arch Otorhinolaryngol [Internet]. 2006 Jun [cited 2016 Nov 30];263(6):586-91.

12. Pai SA, Naresh KN, Masih K, Ramarao C, Borges AM. Teratocarcinosarcoma of the paranasal sinuses: a clinicopathologic and immunohistochemical study. Hum Pathol [Internet]. 1998 Jul [cited 2016 Nov 30];29(7):718-22.

13. Shindo ML, Stanley RB, Kiyabu MT. Carcinosarcoma of the nasal cavity and paranasal sinuses. Head Neck [Internet]. [cited 2016 Nov 30];12(6):516-9.

\section{How to cite this article?}

Shaikh S, Fernandes G, Rojekar A, Joshi A. Sinonasal Teratocarcinosarcoma. Int J Med Res Rev 2016;4(12):21812185.doi:10.17511/ijmrr. 2016.i12.17. 